\title{
Efectos de la rehabilitación energética sobre las primeras torres residenciales de Gipuzkoa (1958-1974). Notas para los arqueólogos del futuro
}

\author{
Effects of the energy rehabilitation on the first residential towers in \\ Gipuzkoa (1958-1974). Some notes for the archaeologists of the future
}

L. Etxepare $^{(*)}$, E.J. Uranga ${ }^{(*)}$, M. Sagarna ${ }^{(*)}$, I. Lizundia ${ }^{(*)}$

RESUMEN

Tras la promulgación de la Ley del Suelo (1956) y durante un ciclo que habría de durar cerca de veinte años, fueron construidas las primeras torres residenciales en el territorio de Gipuzkoa. Del estudio de una serie de casos representativos, se obtiene que los arquitectos guipuzcoanos ensayaron en ellas una serie de soluciones específicas, caracterizadas por el empleo de diversos materiales y texturas que evidenciaban su lógica constructiva y dotaban a las fachadas de una especial materialidad. Medio siglo más tarde, como consecuencia de la rehabilitación energética, estas envolventes están siendo objeto de un proceso de cobertura que se expande con rapidez. El presente artículo procede a analizarlas antes de que desaparezcan complemente de nuestra vista, levantando acta de sus características constructivas, estructurales y compositivas.

Palabras clave: Rehabilitación energética, primeras torres residenciales, años 60 del siglo XX, ocultación de fachadas originales.

\section{ABSTRACT}

After the enactment of the Land Law (1956) and during a cycle that lasted nearly 20 years, the first residential towers were constructed in Gipuzkoa, one of the territories of the Basque Autonomous Community, in Spain. As a result of the study of a series of representative cases, there is obtained that the architects of Gipuzkoa made use of them in order to test specific solutions. These solutions were characterized by the employment of diverse materials and textures that demonstrated the constructive logic of this new architectural type, providing to the façades of a special materiality. Half a century later, as a consequence of the energy refurbishment, these building envelopes are experiencing a process of coverage that expands with rapidity. The present article proceeds to analyze them before they disappear, drawing up a record of their constructive and structural characteristics, as well as their composition style.

Keywords: Energy refurbishment, first residential towers, 1960s, original façade covering.

(*) Departamento de Arquitectura. Universidad del País Vasco / Euskal Herriko Unibertsitatea.

Persona de contacto/Corresponding author: lauren.etxepare@ehu.eus (L. Etxepare).

ORCID: https://orcid.org/oooo-0oo2-9849-1244 (L. Etxepare); https://orcid.org/oooo-0oo2-3416-5511 (E.J. Uranga); https://orcid.org/oooo-0001-7409-030X (M. Sagarna); https://orcid.org/oooo-0001-7336-1083 (I. Lizundia)

Cómo citar este artículo/Citation: Etxepare, L.; Uranga, E.J.; Sagarna, M.; Lizundia, I. (2019). Efectos de la rehabilitación energética sobre las primeras torres residenciales de Gipuzkoa (1958-1974). Notas para los arqueólogos del futuro. Informes de la Construcción, 71(555): e304. https://doi.org/10.3989/ic.64632.

Copyright: (C) 2019 CSIC. Este es un artículo de acceso abierto distribuido bajo los términos de la licencia de uso y distribución Creative Commons Reconocimiento 4.0 Internacional (CC BY 4.0). 


\section{INTRODUCCIÓN}

Como consecuencia de la rehabilitación energética basada en la adición de nuevos sistemas por el exterior de la envolvente vertical, están quedando ocultas una serie de soluciones históricamente empleadas en las fachadas de los edificios residenciales. Es un hecho que está afectando especialmente a aquellos edificios que no cuentan con ningún grado de protección y en cuya rehabilitación no está siendo considerada la preservación de la composición original. Esta manera de proceder, por otro lado, no afecta por igual a todos los lenguajes arquitectónicos; son precisamente aquellas obras en cuyas fachadas se optó por acentuar la materialidad y por manifestar su lógica constructiva, las que en mayor grado están resultando desvirtuadas.

El presente artículo estudia la incidencia del fenómeno sobre la envolvente de las primeras torres residenciales de los años 60 , cuya composición original, caracterizada por el empleo de diversos materiales y texturas, está llamada a desaparecer. Para ello, ha sido adoptado un conjunto de casos representativos edificados en el territorio de Gipuzkoa durante un ciclo que se inició tras la promulgación de la Ley del Suelo (1956) y que remitió a mediados de la década de los 70, en un contexto de crisis económica y de cambio de régimen político. A modo de notas para los arqueólogos del futuro, tarde ya para que una posible política de protección salvaguarde estas fachadas y antes de que desaparezcan por completo de nuestra vista, los autores proceden a analizarlas, levantando acta de sus características desde un punto de vista integral, en el que la lógica constructiva, estructural y compositiva resultan indisociables.

\section{CASOS DE ESTUDIO: LAS PRIMERAS TORRES DE VIVIENDA EN GIPUZKOA (1958-1974)}

En los años sesenta del siglo veinte, como consecuencia de la fuerte inmigración y debido también al crecimiento vegetativo, el incremento de la población del País Vasco llegó a ser el doble que en décadas anteriores: si con anterioridad a 1950 la población experimentaba un crecimiento medio de un $15 \%$ por década, a partir de entonces la tasa llegó a duplicarse. En 1970, la población de Gipuzkoa alcanzaba los 630.000 habitantes, y en respuesta a la urgencia de nuevas viviendas, la gestión urbanística vino a dotarse de herramientas más eficaces para el desarrollo del planeamiento local, como el Plan de Ordenación Territorial de Guipúzcoa (1944) y los Planes Generales de Ordenación de Tolosa (1954), Rentería (1955) o Eibar (1959).

En el ámbito estatal, se ponía en marcha el primer Plan Nacional de Vivienda (1955), entre cuyos objetivos estaba el de construir 550.00o viviendas amparadas en la Ley de viviendas de Renta limitada. Más tarde, en 1959, el Gobierno aprobaba el Plan de Estabilización; de su mano llegaría el Plan de Desarrollo (1964-1967), cuyo objetivo consistía en elevar al máximo la tasa de crecimiento del Producto interior Bruto. Gracias a estos planes locales y estatales, y por impulso del Instituto Nacional de la Vivienda, de particulares, cooperativas y promotoras municipales, la edificación de viviendas creció como nunca lo había hecho antes: $\mathbf{1 1 . 5 0 0}$ edificios que albergan cerca de 100.000 viviendas, el $22 \%$ del parque guipuzcoano actual, fueron construidos entre 1961 y 1980 (1). Dicho crecimiento se manifestaría también en los tipos residenciales adoptados, cuyo perfil edificatorio tendió a aumentar.

\subsection{Ensayos sobre un nuevo tipo edificatorio}

Más determinante aún que los Planes de Ordenación y los Planes Nacionales, fue la entrada en vigor de la Ley del Suelo de 1956, que implantaba un análisis integral del territorio mediante figuras de planeamiento de diversa escala: el Plan Provincial, el Plan General de Ordenación Urbana y el Plan Parcial (2). Una de las herramientas novedosas que dispuso la Ley, habida cuenta de su competencia para convertir el suelo de reserva en suelo urbano, fue el Polígono, cuya creación constituía el primer paso para la construcción de futuros barrios. El desarrollo del Polígono correspondía a la figura denominada Plan Parcial, quien definía todas sus características urbanísticas, como las alineaciones, alturas, volumen y aprovechamientos (3). Esta figura resultó primordial a la hora de ensayar los nuevos tipos residenciales. Entre ellos adquirió un especial protagonismo el Polígono compuesto de una serie de torres, si bien el calificativo de torre vendría dado no tanto por la altura que alcanzaban sino por las proporciones que adoptaban; al fin y al cabo, a pesar de que la más alta de ellas alcanzara las 22 plantas, la altura media era de 14. A partir de entonces, el polígono formado por un conjunto de torres se convirtió en una solución recurrente que sería llevada a cabo en no pocos municipios guipuzcoanos. Consistía en una solución rentable, dado el aprovechamiento del suelo que proporcionaba, gracias al cual las clases medias pudieron acceder a una vivienda en propiedad, incluso en lugares de veraneo hasta la fecha reservados a las élites aristocráticas (4).

La implantación del nuevo tipo residencial se desarrolló durante un ciclo de casi 20 años, remitiendo con la crisis del petróleo (1974-79). Por modesta que sea la realidad que el conjunto de casos de estudio representa, su análisis prueba la reproducción local de un fenómeno que se dio en todo el mundo capitalista, consecuencia de la correlación entre los ciclos económicos y la evolución de los tipos edificatorios: es precisamente cuando los tipos se intensifican y optan por la altura en forma de rascacielos, torres y otros modelos esbeltos, cuando se vislumbra la antesala de la crisis que provoca el fin de un ciclo económico (5). A mediados de los 70, la evolución demográfica en Bizkaia y Gipuzkoa entraba en declive (6), y el ritmo de edificación de vivienda se reducía a la cuarta parte del de la década anterior. Si en 1980, la vivienda libre terminada en la Comunidad Autónoma Vasca suponía el 30\% de la edificada, al año siguiente pasaba a ser el 21\%, cayendo hasta el 10\% en el año 1983 (7). Hasta 1988, la edificación de vivienda sería liderada por el Gobierno Vasco, quien adoptaría otros modelos tipológicos de menor intensidad, en coherencia con la estabilización de la evolución demográfica.

\subsection{Especificidad de los casos: Innovación tipológica versus continuismo tecnológico}

El nuevo tipo arquitectónico nacía con una contradicción característica de la arquitectura de los países en vías de desarrollo: si bien los primeros casos denotaban una voluntad de innovación en todo lo relativo al tipo edificatorio, su construcción no vino acompañada de significativos avances tecnológicos. Sirva el análisis de las 15 plantas tipo aportadas como muestra de la primera de las características (ver figuras 1,2 y 3). Se trata de un conjunto plural en lo que a la solución en planta se refiere, fruto de una investigación inspirada por los atlas publicados en Europa y Estados Unidos, basada en parámetros relacionados con las necesidades del habitante: la superficie, el soleamiento y la correcta relación entre las es- 
tancias (8). Sin embargo, en lo que a la construcción se refiere, y especialmente en lo relativo a la envolvente, el conjunto de casos da fe de la característica mutación que la tecnología de la fachada experimentó en España, origen de una solución constructiva que no se reprodujo en otros países europeos.

Hasta finales del siglo XIX, en Europa, la construcción en ladrillo había venido caracterizándose por el carácter estructural de sus muros y por la composición homogénea de estos (9). Entretanto, en Gran Bretaña, se gestaba el cavity wall para la construcción masiva de viviendas que albergaran a la creciente población de los núcleos industriales. Por primera vez, la fachada de ladrillo quedaba disociada en dos hojas, recayendo la función portante de los forjados sobre la hoja interior y encargándose la exterior de formar una cámara, con el fin de dotar a los edificios de una mayor protección frente a la humedad. La solución se extendió por los países centroeuropeos a medida que avanzaba el siglo veinte; no así en España, donde los agentes se decantarían por emplear una hoja portante, que a mediados de siglo comenzaría a trasdosarse con un tabique interior para la formación de una cámara. Cabe mencionar como ejemplo del primer estadio de esta variante, los conjuntos de viviendas sociales de Zaragoza proyectados a partir de 1945 y construidos mediante fachadas de entre medio pie y pie y medio, la mayoría de las cuales trabajaban como muros portantes (10). Sirvan, por otra parte, las viviendas de Usera de 1942 y las viviendas de Virgen del Pilar en 1945-48, en Madrid, proyectadas por Francisco de Asís Cabrero, como ejemplo de los primeros conjuntos residenciales con fachada trasdosada (11).

Tras la Segunda Guerra Mundial (1939-1945), durante la reconstrucción de Europa, la edificación de vivienda adoptó un carácter industrializado, y a medida que las estructuras en acero laminado y hormigón armado favorecían la edificación en altura, el cerramiento de los edificios fue desprovisto definitivamente de toda misión estructural, sustituyéndose el ladrillo por otros componentes prefabricados de mayor tamaño. La prefabricación experimentó un gran auge en Gran Bretaña, con patentes de prefabricados de hormigón, como Cornish Units y Reema Hollow Panel (12); en Alemania occidental, donde buena parte de la vivienda pública fue construida mediante el sistema prefabricado Plattenbauten, y en países nórdicos como Suecia, donde el "One Million Apartment Programme” (1964) fue llevado a cabo mediante sistemas en hormigón prefabricado (13). En Francia, durante la reconstrucción del país tras la Segunda Guerra Mundial, el Ministère de la Reconstruction et de l'Urbanism llevó a cabo una transformación integral del sector de la edificación (14), promoviendo la invención de nuevas patentes, entre las que destacan el sistema Camus o los paneles Mopin (15).

También España participó de dicho impulso: el ingeniero Eduardo Torroja, Director del Instituto Técnico de la Construcción y del Cemento, convocaba en 1949 un Concurso Internacional de Industrialización de Viviendas, con el fin de impulsar la prefabricación y obtener un prototipo de vivienda que se pudiera producir en serie. Esta voluntad de industrializar la construcción seguiría vigente en décadas posteriores, tal como muestran los siguientes dos casos: la construcción mediante paneles prefabricados de los bloques residenciales del polígono de Bellvitge (1964) (16), y el Polígono de Ocharcoaga (1960-64), Bilbao, en uno de cuyos bloques fue empleado el sistema de origen francés Fiorio, que resolvía fachadas completas incorporando ventanas y acabados (17). Mediada la segunda mitad del siglo, el foco se trasladaría de la pre- fabricación a la concepción de la vivienda como un módulo optimizado para ser repetido y apilado espacialmente (18).

Sin embargo, a diferencia de lo que sucedió en los países anteriormente referidos, los sistemas industrializados no terminaron de calar ni de trascender al tejido productivo español, que en la gran mayoría de los casos continuó confiando el cerramiento a una doble hoja cerámica compuesta por un tabique interior, una cámara de aire y una fábrica exterior portante, de un pie o de pie y medio de espesor. La solución perduró incluso a partir de mediados del siglo veinte, tras invertirse la relación que fachada y forjados habían mantenido históricamente, y pasaran a ser los forjados los que sujetaran la fachada (19). El ladrillo macizo sería sustituido por un ladrillo hueco, y todos los agentes de la construcción convendrían en adoptar la solución. La evolución de la envolvente térmica de la vivienda social de Sevilla entre 1939 y 1979, refleja la incorporación de esta solución a mediados de la década de los 50, así como su posterior consolidación (20). Fue tal su expansión, que no sólo se ganó el apelativo de fachada convencional, sino que muchos emplearían el término de fachada tradicional para referirse a ella, sin que respondiera en realidad a ninguna tradición histórica.

No obstante, a pesar de lo rudimentario del sistema, o debido a ello quizá, las fachadas en ladrillo supusieron una invitación a la experimentación; más aún a partir de la irrupción del ladrillo cara vista, y especialmente en la construcción de las primeras torres residenciales: la disposición exenta de estas, su insoslayable presencia urbana y el carácter unitario de su envolvente, que no entiende de fachadas principales, alzados posteriores ni patios de luces, hicieron que la construcción de sus fachadas respondiera a un concepto integral, sistemáticamente empleado en toda su extensión. A diferencia de las soluciones más recatadas empleadas en la construcción del periodo autárquico, la relación entre los paños de ladrillo y los elementos estructurales vistos llegaría a ser objeto de una elaborada composición, que trataba de contrarrestar la carencia de nuevos sistemas industrializados: postes, pantallas y cantos de forjado serían exhibidos sin rubor con el fin de acentuar la potencia de la estructura, cuya misión, tratándose de torres, resultaba más comprometida que lo que había venido siendo hasta entonces. Señalemos, por último, la falta de reparos en rasgar la envolvente del edificio con elementos estructurales, dada la indiferencia que los arquitectos y el sector de la edificación mostraban por los aspectos térmicos, pendientes de normalizar por aquel entonces (ver relación entre cerramiento y estructura en figuras 1,2 y 3 ).

\subsection{Presentación de los casos}

El campo de estudio lo componen las torres residenciales construidas en el territorio de Gipuzkoa entre 1958 y 1974, de las que ha sido establecida una muestra de 15 casos representativos (tabla 1) en base a los siguientes criterios: el conjunto abarca todas las soluciones de fachada empleadas en el periodo objeto de estudio, proporciona un seguimiento continuado de la evolución sin más lagunas temporales que aquellos años en los que no consta la construcción de ninguna torre, y comprende, finalmente, todos los casos que han sido objeto de rehabilitación energética. Han sido ordenadas en función de la fecha de proyecto, especificándose el número de torres correspondiente a la promoción, el municipio, el número de viviendas, el tipo de promotor, los acabados y la participación o no de elementos estructurales en la fachada. 


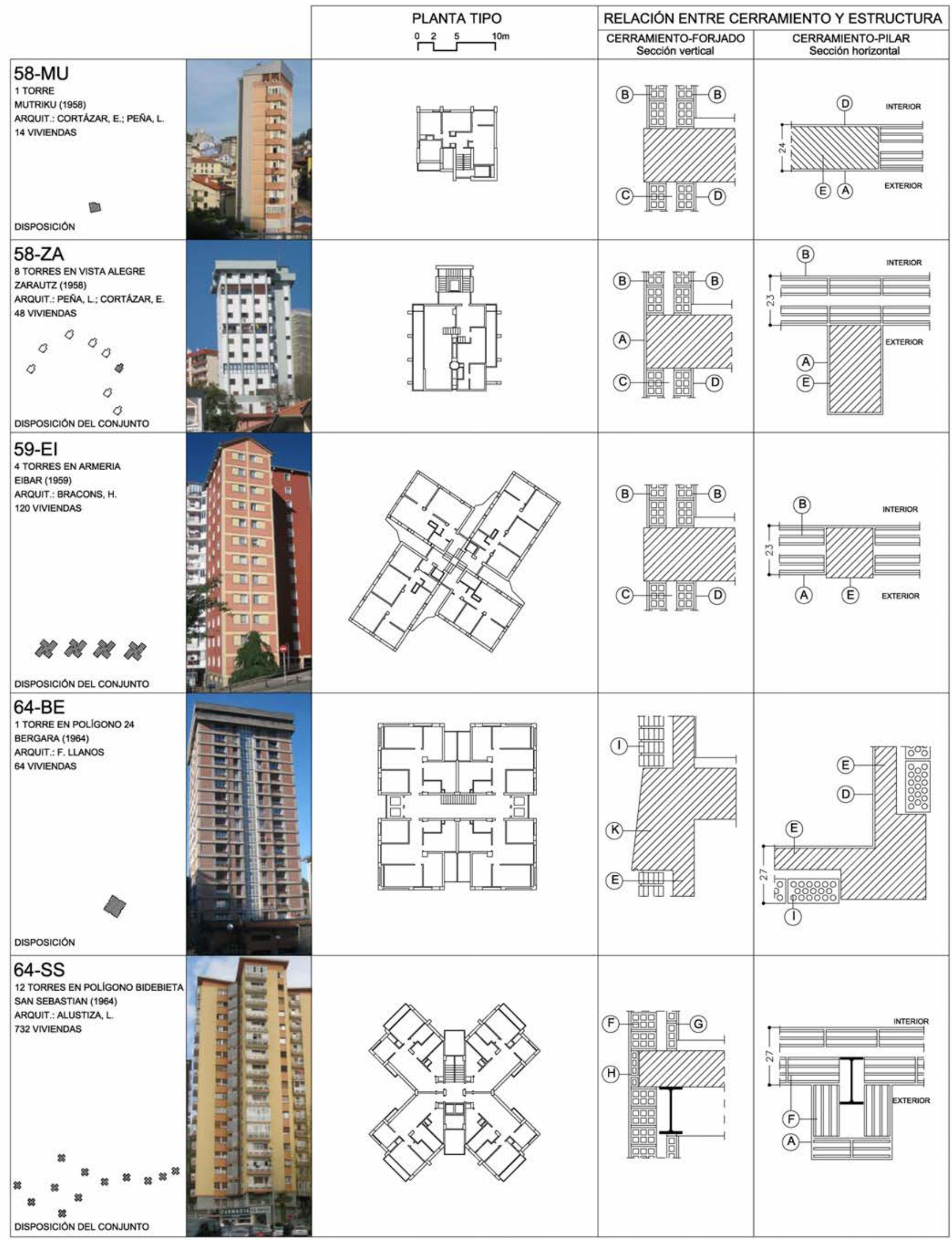

(A) Enfoscado de mortero de cemento pintado (B) Tabicón de ladrillo hueco doble (C) Cámara de aire (D) Enlucido de yeso (E) Poste de hormigón armado F Media asta de ladrillo hueco doble (G)Tabique de ladrillo hueco sencillo (H) Plaqueta cerámica hueca (1) Ladrillo caravista cerámico (J) Raseo de mortero hidrófugo (K) Pantalla de hormigón armado (L) Plaqueta cerámica caravista (M) Plaqueta cerámica caravista a sardinel (N) Faldón colgado de hormigón armado

Figura 1. Casos de estudio (1958-64). 


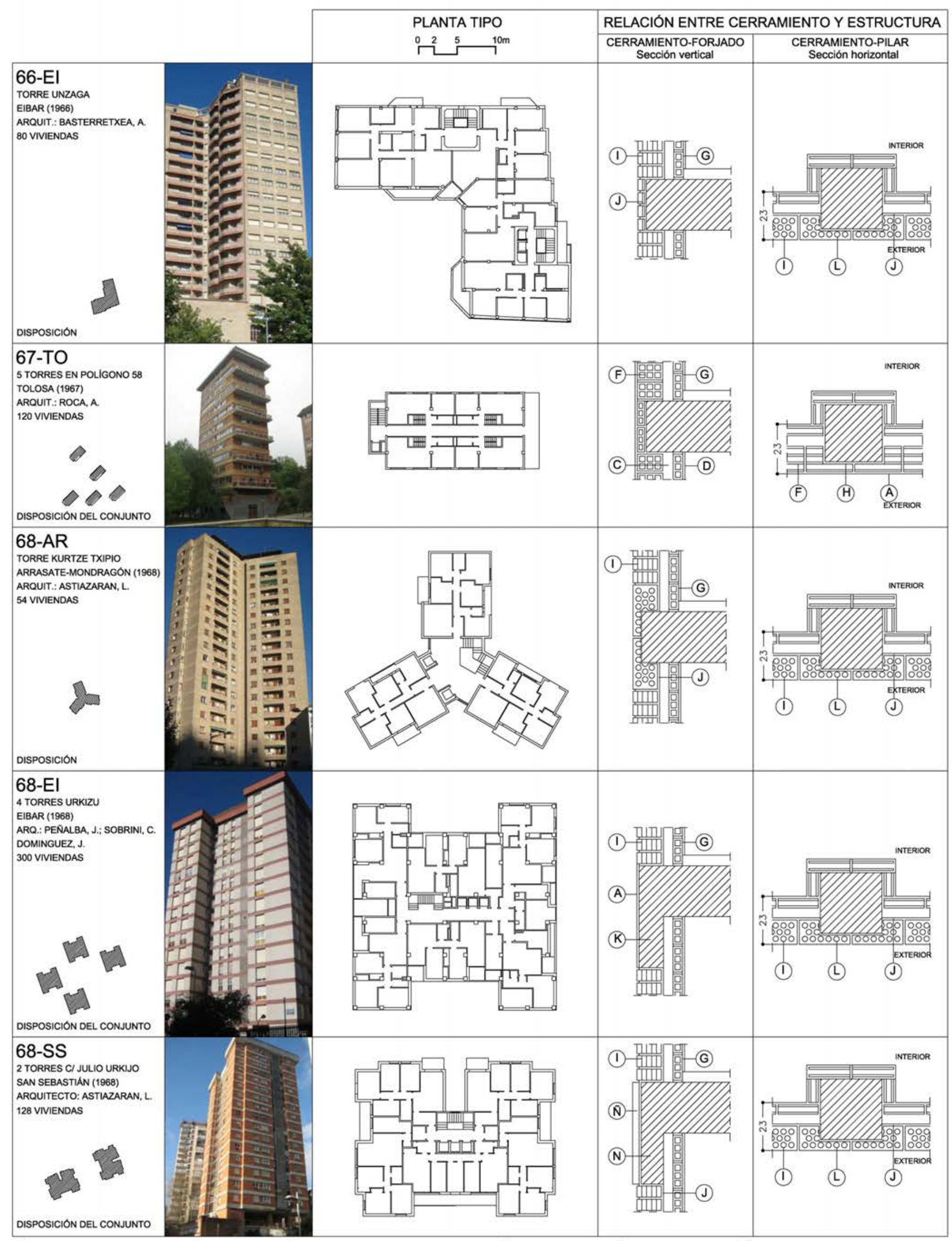

(A) Enfoscado de mortero de cemento pintado (B) Tabicón de ladrillo hueco doble (C) Cámara de aire (D) Enlucido de yeso (E) Poste de hormigón armado F) Media asta de ladrillo hueco doble (G)Tabique de ladrillo hueco sencillo (H) Plaqueta cerámica hueca (1) Ladrillo caravista cerámico (J) Raseo de mortero hidrófugo (K) Pantalla de hormigón armado (L) Plaqueta cerámica caravista (M) Plaqueta cerámica caravista a sardinel (N) Faldón colgado de hormigón armado (N) Plaqueta de piedra adherida con mortero cola

Figura 2. Casos de estudio (1966-68). 


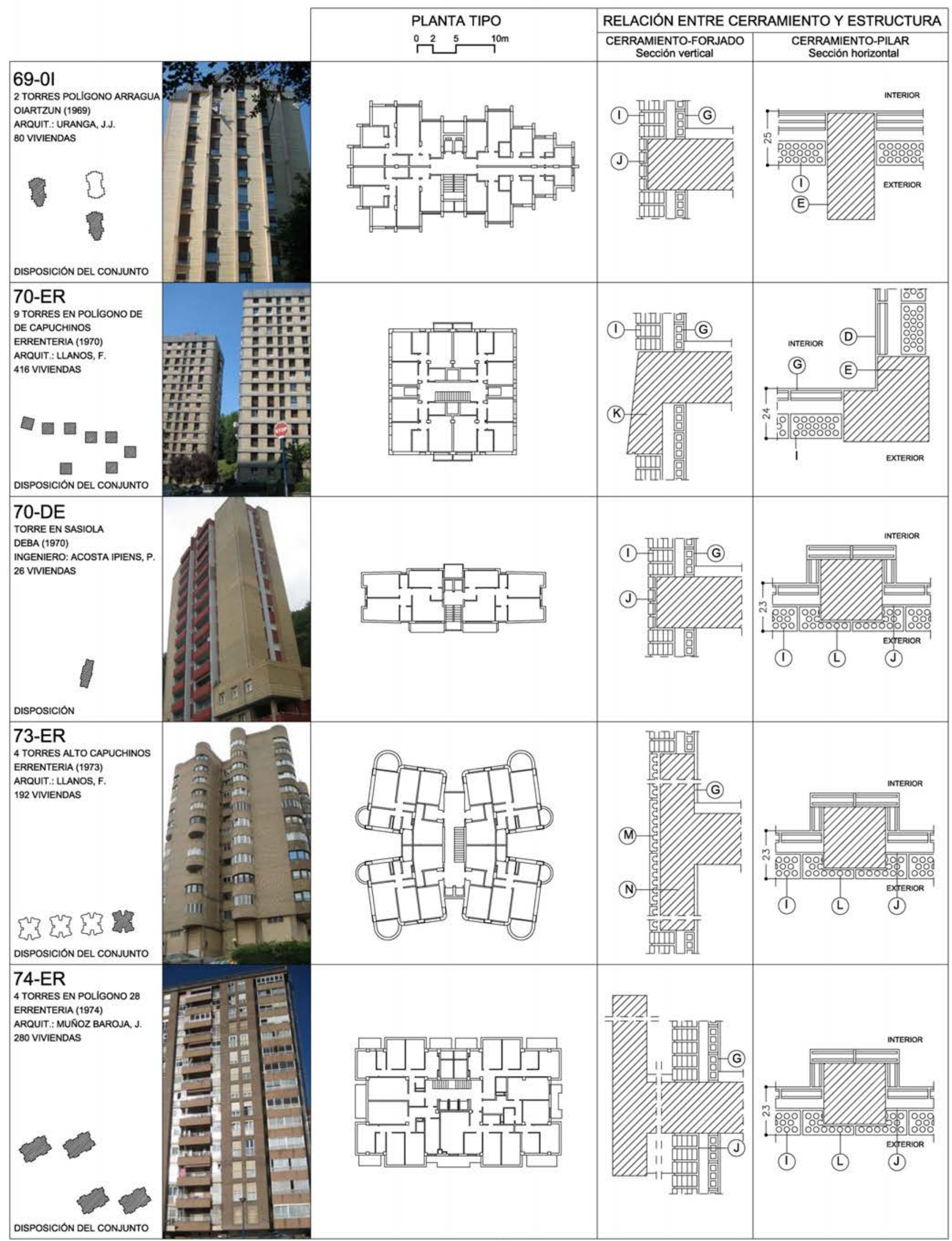

(A) Enfoscado de mortero de cemento pintado (B) Tabicón de ladrillo hueco doble (C) Cámara de aire (D) Enlucido de yeso (E) Poste de hormigón armado (F) Media asta de ladrillo hueco doble (G) Tabique de ladrillo hueco sencillo (H) Plaqueta cerámica hueca (1) Ladrillo caravista cerámico (J) Raseo de mortero hidrófugo (K) Pantalla de hormigón armado (L) Plaqueta cerámica caravista (M) Plaqueta cerámica caravista a sardinel (N) Faldón colgado de hormigón armado 
Tabla 1. Relación de casos de estudio.

\begin{tabular}{|c|c|c|c|c|c|c|c|c|}
\hline Código & Año & Municipio & $\mathbf{N}^{\mathbf{o}}$ & Proyecto & No Viv. & $\begin{array}{l}\text { Prom. } \\
\text { (1) }\end{array}$ & $\begin{array}{c}\text { Acab. } \\
\text { (2) }\end{array}$ & $\begin{array}{l}\text { Estruc. } \\
\text { (3) }\end{array}$ \\
\hline 58-MU & 1958 & Mutriku & 1 & Torre en el puerto & 14 & $\mathrm{M}$ & $\mathrm{RC}$ & $\sqrt{ }$ \\
\hline $58-\mathrm{ZA}$ & 1958 & Zarautz & 8 & Torres Vista Alegre & 48 & $\mathrm{P}$ & RA & $\sqrt{ }$ \\
\hline 59-EI & 1959 & Eibar & 4 & Torres en Armeria & 120 & $\mathrm{M}$ & $\mathrm{RC}$ & \\
\hline $64-B E$ & 1964 & Bergara & 1 & Torre en Polígono 24 -Zabalotegui- & 64 & $\mathrm{M}$ & $\mathrm{CV}$ & $\sqrt{ }$ \\
\hline 64-SS & 1964 & San Sebastián & 12 & Torres en Polígono de Bidebieta & 732 & INV & $\mathrm{RC}$ & \\
\hline 66-EI & 1966 & Eibar & 1 & Torre Unzaga & 24 & $\mathrm{P}$ & $\mathrm{CV}$ & \\
\hline $67-\mathrm{TO}$ & 1967 & Tolosa & 5 & Torres en Polígono 58 & 60 & $\mathrm{C}$ & $\mathrm{RC}$ & \\
\hline 68-AR & 1968 & Arrasate & 1 & Torre Kurtze-Txipio & 54 & $\mathrm{P}$ & $\mathrm{CV}$ & \\
\hline 68-SS & 1968 & San Sebastián & 2 & Torres en C/ Julio Urkijo & 128 & $\mathrm{P}$ & $\mathrm{CV}$ & $\sqrt{ }$ \\
\hline 68-EI & 1968 & Eibar & 4 & Torres Urkizu & 360 & $\mathrm{P}$ & $\mathrm{CV}$ & $\sqrt{ }$ \\
\hline 69-OI & 1969 & Oiartzun & 2 & Torres Polígono Oyarzun-Rentería & 80 & $\mathrm{P}$ & $\mathrm{CV}$ & $\sqrt{ }$ \\
\hline 70-ER & 1970 & Errenteria & 9 & Torres en Polígono de Capuchinos & 480 & $\mathrm{P}$ & $\mathrm{CV}$ & $\sqrt{ }$ \\
\hline $70-\mathrm{DE}$ & 1970 & Deba & 1 & Torre en Sasiola & 26 & GC & $\mathrm{CV}$ & $\sqrt{ }$ \\
\hline 73-ER & 1973 & Errenteria & 6 & Torres en el alto de Capuchinos & 336 & $\mathrm{P}$ & $\mathrm{CV}$ & $\sqrt{ }$ \\
\hline 74-ER & 1974 & Errenteria & 4 & Torres en Polígono 28 & 280 & $\mathrm{P}$ & $\mathrm{CV}$ & $\sqrt{ }$ \\
\hline
\end{tabular}

(1) Promotor: (M) Municipal (P) Particular (C) Cooperativa (INV) Instituto Nacional de la Vivienda (GC) Guardia Civil.

(2) Acabado: (RC) Revestimiento contínuo (RA) Revestimiento adherido (CV) Ladrillo caravista.

$(3)$ Estructura: $(\sqrt{ })$ Presencia de elementos estructurales con vocación compositiva.

\section{EL FENÓMENO DE LA REHABILITACIÓN ENERGÉTICA}

La primera norma española que establecía la justificación de la envolvente desde el punto de vista térmico fue la NBECT-79, que entraba en vigor cinco años después de que fuera construido el último y más reciente de los casos de estudio (74-ER). Sólo a partir de entonces comenzaría a incorporarse una capa aislante a los cerramientos. El ámbito de aplicación de la norma, no obstante, se limitaba a la obra nueva. En realidad, los antecedentes de la rehabilitación energética del parque residencial español apenas se remontan a los primeros años del presente siglo, cuando fue aprobada la Directiva europea 2002/91/CE, relativa a la eficiencia energética de los edificios, que consideraba las obras de rehabilitación como una oportunidad para reducir los consumos de los edificios. Su transposición dio como resultado la aprobación en 2006 del Código Técnico de la Edificación (CTE), con su Documento Básico DB-HE sobre el Ahorro de Energía en la edificación. Este documento sería de aplicación no sólo en los edificios nuevos, sino en aquellos existentes que contaran con una superficie útil superior a $1.000 \mathrm{~m}^{2}$, en los que se acometiera la reforma de una cuarta parte de su envolvente.

La siguiente Directiva europea en aprobarse fue la 2010/31/ UE. En la misma se establecía la mejora de la eficiencia energética de los edificios existentes como objetivo a alcanzar en el año 2020. Una vez transpuesta esta Directiva, fueron aprobados en España la Orden FOM/1635/2013 de actualización del DB-HE, y el nuevo R.D. 235/2013 sobre la Certificación de Eficiencia Energética, que establecía un Certificado de Eficiencia Energética para los edificios existentes que fueran a ser vendidos o alquilados. Posteriormente se aprobó la Directiva 2012/27/UE, en la que se instaba a los Estados a incidir en la mejora energética del parque edificado existente y en establecer una estrategia a largo plazo. Como consecuencia de ello fue aprobada en el 2013 una ley fundamental para poder emprender con ambición la rehabilitación energética del parque residencial: la Ley 8/2013 de Rehabilitación, Regene- ración y Renovación Urbanas. En la misma se preveía que las futuras intervenciones sobre los edificios residenciales iban a resultar fundamentales para la mejora de la eficiencia energética.

\subsection{El viraje del sector hacia la rehabilitación energética}

La nueva visión de la eficiencia energética hacía entrada en un momento en el que sobre la edificación de obra nueva y la economía española se cernía el gran colapso provocado por el pinchazo de la burbuja inmobiliaria, consecuencia del cual, entre 2007 y 2010, la construcción de edificios residenciales de nueva planta cayó cerca de un 90\% (21). Dado que buena parte de la economía y del PIB estatal había venido dependiendo del sector de la construcción y ante la certeza de que la edificación de nueva planta no iba a recuperar en años los niveles anteriores de actividad, la misión de reactivar la economía del sector recayó sobre la rehabilitación de vivienda. La eficiencia energética no sólo pasó a ser incorporada a las políticas de rehabilitación, sino que se convirtió en el eje central del sector, no siendo posible plantear la reparación de la envolvente de un edificio residencial sin mejorar considerablemente su nivel de aislamiento térmico.

Tras la promulgación de la Ley 8/2013, que establecía la colaboración y la cooperación económica de la Administración General del Estado, los gobiernos autonómicos y la administración local, fueron aprobados diferentes programas con el objeto de gestionar las ayudas públicas: en el ámbito estatal, de la mano del Instituto para la Diversificación y Ahorro de la Energía, fue puesto en marcha el Programa de ayudas a la rehabilitación energética de edificios en el ámbito estatal (PAREER-CRECE); en el marco autonómico, en lo que a Euskadi se refiere, el Ente Vasco de la Energía ponía en marcha el Plan RENOVE, y en el ámbito provincial, las diputaciones forales posibilitaban que los propietarios pudieran deducir fiscalmente en la Declaración de la Renta de las Personas Físicas la inversión realizada. A escala municipal, dependiendo 
en parte del perfil político de los gobiernos municipales y de la situación financiera de las entidades locales, fueron concedidas subvenciones para la rehabilitación de vivienda, que adoptaron el rango de "ordenanza reguladora".

\subsection{La rehabilitación energética y su colisión con la preservación}

Dejando a un lado la intervención sobre los sistemas de agua caliente sanitaria, climatización e iluminación, la medida más efectiva para hacer más eficiente un edificio residencial, consiste en limitar su demanda energética, para lo cual resulta fundamental reducir la transmitancia de las distintas partes de las que se compone su envolvente. En lo que a los paños ciegos se refiere, son dos, como mínimo, los tipos de soluciones: la incorporación de una capa aislante por el intradós de la fachada, y la adhesión de un aislamiento por su parte exterior, ya sea acabado con un revoco, como es el caso de los Sistemas de Aislamiento Térmico por el Exterior (SATE), o mediante una fachada ventilada. Cabe, además, en el caso de las fachadas de doble hoja cerámica, una tercera vía, consistente en la inyección de espuma de poliuretano o de celulosa en el interior de la cámara.

No obstante, en lo que al conjunto de torres analizadas respecta, del "Estudio del impacto en el coste de la rehabilitación de la vivienda construida entre 1960 y 1980 en el País Vasco" (22), llevado a cabo por los autores, se obtiene que, de entre las tres soluciones, es aquella basada en la actuación por el extradós la que se está imponiendo, dada una serie de motivos de peso: la continuidad de la nueva envolvente incorporada, la eliminación de los puentes térmicos, y el hecho de trabajar por el exterior del edificio, salvaguardando el interior de las estancias.

A la luz de estos casos, la vía de mejora de la envolvente que más éxito está teniendo desde la entrada en vigor del CTE, es, precisamente, aquella que colisiona con la preservación del carácter original de las fachadas. Dado el impulso adquirido por esta modalidad, la parte del parque residencial no protegida está experimentando un veloz proceso de muda, con la consiguiente transformación del paisaje urbano (ver figura 4).

\subsection{Soluciones empleadas en los casos de estudio}

Cinco de los 15 casos expuestos en las figuras 1, 2 y 3, han sido objeto de rehabilitación en los últimos años (ver figura 4). Se exponen a continuación las soluciones empleadas, aportando una descripción de la solución, y cuantificando la transmitancia térmica del paramento ciego de fachada en su estado original $\left(\mathrm{U}_{\mathrm{o}}\right)$, y una vez rehabilitado $\left(\mathrm{U}_{\mathrm{r}}\right)$. La reducción alcanzada, en los cinco casos, es de cerca de un 70\%, haciendo que se cumpla lo establecido por el CTE, que en la zona climática $\mathrm{D}$, asigna a los muros una transmitancia térmica máxima de $\mathrm{o}, 60 \mathrm{~W} / \mathrm{m}^{2} . \mathrm{K}$.

- 68-SS. Aislamiento de lana mineral (e=50 mm, l=0,038 W/ (m.K)), fijado a la fachada original, subestructura de montantes, ménsulas y piezas de cuelgue en aluminio, cámara de aire $(4 \mathrm{~cm})$, y placas cerámicas extrusionadas alveolares de $39 \mathrm{~mm}$ espesor, $400 \mathrm{~mm}$ de altura y longitud variable. $\mathrm{U}_{\mathrm{o}}: 1,55 \mathrm{~W} / \mathrm{m}^{2} . \mathrm{K} \rightarrow \mathrm{U}_{\mathrm{r}}: 0,51 \mathrm{~W} / \mathrm{m}^{2} . \mathrm{K}$.

- 69-OI. Plancha de poliestireno expandido ( $=60 \mathrm{~mm}$, $\mathrm{l}=0,04 \mathrm{~W} /(\mathrm{m} . \mathrm{K}))$, adherida mediante mortero y fijada con anclajes a la fachada original, revestida con mortero armado de malla, y acabada con mortero acrílico y pintura. $\mathrm{U}_{\mathrm{o}}$ : $1,55 \mathrm{~W} / \mathrm{m}^{2} . \mathrm{K} \rightarrow \mathrm{U}_{\mathrm{r}}: 0,47 \mathrm{~W} / \mathrm{m}^{2} . \mathrm{K}$.

- 70-ER. Subestructura de montantes en acero galvanizado, aislamiento de lana mineral (45 mm, l=0,038 W/(m.K)), barrera de agua Tyvek, cámara de aire de espesor variable y panel de acabado con alma de cemento portland con aditivos y material aligerante, recubierto por una malla de fibra de vidrio y pintura. $\mathrm{U}_{\mathrm{o}}: 1,55 \mathrm{~W} / \mathrm{m}^{2} . \mathrm{K} \rightarrow \mathrm{U}_{\mathrm{r}}: 0,54 \mathrm{~W} / \mathrm{m}^{2} . \mathrm{K}$.

- 73-ER. Subestructura de montantes en acero galvanizado, aislamiento de lana mineral ( $45 \mathrm{~mm}, \mathrm{l}=0,038 \mathrm{~W} /(\mathrm{m} . \mathrm{K})$ ), barrera de agua Tyvek, cámara de aire $(4 \mathrm{~cm})$ y panel de acabado con alma de cemento portland con aditivos y material aligerante, recubierto por una malla de fibra de vidrio y pintura. $\mathrm{U}_{\mathrm{o}}: 1,55 \mathrm{~W} / \mathrm{m}^{2} . \mathrm{K} \rightarrow \mathrm{U}_{\mathrm{r}}: 0,54 \mathrm{~W} / \mathrm{m}^{2} . \mathrm{K}$.

- 74-ER. Aislamiento de lana mineral (50 mm, l=0,038 W/ (m.K)), fijado a la fachada original, subestructura de montantes, ménsulas y piezas de cuelgue en aluminio, cámara de aire $(4 \mathrm{~cm})$ y panel plegado compuesto por doble lámina de aluminio y alma de polietileno, de $4 \mathrm{~mm}$ de espesor. $\mathrm{U}_{\mathrm{o}}$ : $1,55 \mathrm{~W} / \mathrm{m}^{2} . \mathrm{K} \rightarrow \mathrm{U}_{\mathrm{r}}: 0,51 \mathrm{~W} / \mathrm{m}^{2} . \mathrm{K}$.

\section{NOTAS PARA LOS ARQUEÓLOGOS DEL FUTURO}

Del análisis de los casos se obtiene que el sistema de cerramiento empleado en la construcción de las primeras torres residenciales en Gipuzkoa experimentó una evolución durante los años en los que se desarrolló el ciclo edificatorio. Esta evolución responde a tres periodos, establecidos en función de la composición del cerramiento, de la participación de la estructura en las fachadas, de la relación constructiva entre ambos elementos, y del empleo o no del ladrillo caravista.

\subsection{Cerramiento de doble tabicón cerámico (1958)}

El cerramiento de las dos primeras torres residenciales de Gipuzkoa, levantadas en los últimos años 50, fue resuelto con un doble tabicón de ladrillo hueco doble y una cámara de aire intermedia. El grosor de la envolvente era de unos 24 $\mathrm{cm}$. La hoja exterior quedaba enteramente apoyada sobre el borde del forjado, y su acabado consistía en un revestimiento continuo (58-MU) o en un revestimiento adherido por el exterior (58-ZA). El caso 58-MU, además, presenta paramentos de hormigón visto, dado que la estructura fue resuelta con pantallas de hormigón que envolvían parcialmente el edificio. En ambos casos, sea de manera enfatizada (58-ZA) o mediante texturas planas (58-MU), la estructura asoma en fachada, combinándose con paños enfoscados y pintados.

\subsection{Consolidación de la fachada convencional (1959-1967)}

En las torres construidas en la primera mitad de la década, el cerramiento fue mayoritariamente resuelto con la solución que mayor éxito tuvo en la España de los años 60: una media asta de ladrillo hueco doble enfoscada y pintada por el exterior, una cámara de aire y un tabique de ladrillo hueco sencillo por el interior, guarnecido y enlucido (67-TO). Si bien es cierto que en algunos casos los postes perimetrales fueron recrecidos y cubiertos con machones de albañilería (64-SS), la estructura quedaba oculta en todos los casos. Consiste en una solución que trata de que el cerramiento sea percibido como un paramento continuo. 


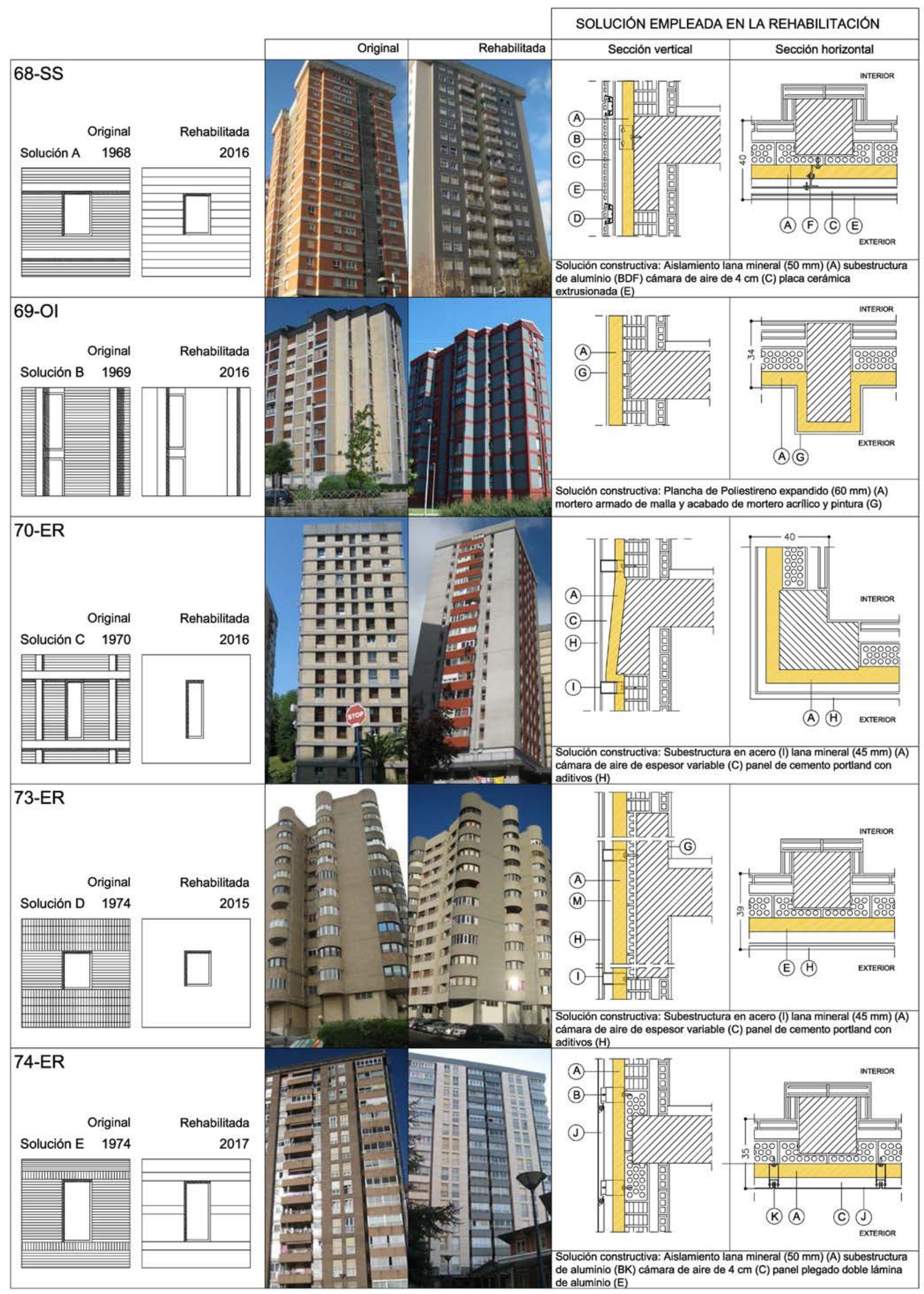

Figura 4. Transformación de la envolvente sobre 5 casos (2015-17). 


\subsection{La irrupción del ladrillo caravista y su connotación cultural (1966-1974)}

A partir de mediados de los 60 irrumpiría con fuerza el ladrillo caravista. Se trataba de un producto de origen natural, cuya producción no requería de un alto nivel de industrialización, y los promotores particulares trataban de ponerlo en valor: era un material apto para ser mostrado, que dotaba a la casa de un valor añadido. Los plazos más largos de colocación del caravista, en relación a la colocación del ladrillo corriente, por otra parte, venían compensados por los tiempos más breves durante los cuales había que mantener los andamios y sistemas auxiliares, dado que no requerían de enfoscado ni de pintura.

Las tejerías guipuzcoanas no elaboraban este tipo de ladrillo, que era suministrado por empresas de Tudela, como en el caso 69-OI, o de otros lugares como Albacete, Palencia, Jaén o Madrid. La longitud de la pieza era, generalmente, de 24 $\mathrm{cm}$, y el espesor variaba entre los 10 y los $12 \mathrm{~cm}$ (23). La hoja exterior quedaba generalmente aparejada a sogas, y la disposición vertical del ladrillo quedaba reservada exclusivamente para chapear elementos en hormigón, como antepechos y petos, que quedaban recubiertos con una plaqueta de $2 \mathrm{~cm}$ de grosor dispuesta a sardinel. En cuanto a la modulación, fue mayor el rigor empleado en aquellos casos en los que la fábrica envolvía ininterrumpidamente el edificio (66-EI y 72DE) y hubo que emplear el ladrillo métrico ( $24 \times 11,5 \times 5)$, que en aquellos casos en los que los paños de ladrillo caravista quedaban completamente interrumpidos por la apertura de un hueco.

No obstante, al margen de las cuestiones constructivas, la irrupción del ladrillo caravista respondía también a otro impulso de tipo cultural, incluso ideológico, relativo a la opción que los arquitectos guipuzcoanos de los años 6o hicieron por el ladrillo visto. Efectivamente, la elección del material se hacía eco de la influencia del movimiento racionalista italiano llamado Tendenza, contrapuesto a la otra corriente neorracionalista basada en el empleo casi exclusivo del color blanco, tan característico en las obras de Peter Eisenman, Michael Graves, Charles Gwathmey, John Hejduk y Richard Meier, conocidos a raíz de la exposición de 1969 en el MoMA de Nueva York con motivo de un simposio de la Conference of Architects for the Study of the Environment (24).

En contraste con las obras de aquellos, también llamados “The New York Five”, arquitectos italianos como Giorgio Grassi y Aldo Rossi, profesaban la necesidad de aunar el pensamiento racionalista de la modernidad con los tipos arquitectónicos históricos (25). Libros como Teoria e storia dell'architettura, de Grassi (1967), traducidos al español por la editorial Gustavo Gili, estaban de candente actualidad a finales de los años 60 (26), y a comienzos de los años 70, la Delegación de San Sebastián del Colegio Oficial de Arquitectos Vasco-Navarro organizaba una serie de jornadas sobre arquitectura, a las que fueron invitados algunos de los arquitectos más representativos del movimiento Tendenza, como Massimo Scolari, Carlo Aymonino, Manfredo Tafuri y el propio Rossi (27). En aquellos encuentros, los italianos defendían el empleo del color natural de los materiales sin revestir, como los mármoles, el hormigón o el ladrillo caravista, con el fin de dotar a las obras de un aspecto monumental y de integrarlas en los tejidos urbanos históricos, y elogiaban la aportación de la sombra, recurso inmaterial que enfatizaba el orden de la fachada y la presencia de los elementos estructurales (ver figura 5). La solución constructiva de estas envolventes incorporaba así un argumento compositivo que no debería ser subestimado.

En cualquier caso, ya formen un conjunto testimonial de carácter tecnológico o de reminiscencias culturales, las diversas soluciones empleadas en los casos de estudio pueden tipificarse en función de la homogeneidad de la fábrica de ladrillo caravista, de la ocultación o no de los elementos estructurales, y del empleo de la plaqueta adherida. Enumeremos a continuación las cinco soluciones obtenidas:

\subsubsection{Solución A: la fachada estratificada}

Fue la solución empleada para poner en evidencia la relación entre forjados y fábrica, que experimentó, a su vez, una evolución: si bien en los años 50 el forjado asomaba a la fachada sin mayor grosor que el de su canto real, en las torres de viviendas guipuzcoanas, el elemento fue concebido con un faldón que colgaba de su borde, y que cumplía a su vez una doble misión: acentuar la estratificación de los pisos, por un lado, y servir como cabezal de los huecos, resueltos mediante el propio faldón, sin necesidad de incorporar dinteles (ver caso 68-EI). En el caso 68-SS, el faldón quedaría recubierto mediante una plaqueta de piedra adherida con mortero cola (ver figura 5).

\subsubsection{Solución B: contrafuertes en hormigón}

Inversamente a la solución anterior, los frentes de forjados quedarían revestidos por hiladas de ladrillo caravista dispuesto a sogas, y serían precisamente los postes perimetrales los que emergerían al exterior, manifestando la solidez y capacidad de la estructura. En ocasiones adoptaban rasgos expresivos, como si de los contrafuertes de una iglesia gótica se tratase. Así sucede en el caso 69-OI, donde los postes perimetrales recorren verticalmente toda la fachada, marcando unas acentuadas sombras que dotan de profundidad a la misma (ver figura 5).

\subsubsection{Solución C: la fábrica confinada}

El Arquitecto Félix Llanos recurrió a la fábrica confinada para resolver la fachada de la torre residencial en ladrillo caravista construida en el Polígono 24 de Bergara (64-BE). Los paños, de una única altura y anchos menores de $2 \mathrm{~m}$, fueron enmarcados en una trama de elementos en hormigón visto y abujardado. Además de los postes perimetrales, quedaban vistos los bordes de los forjados, acentuados mediante un faldón colgado con sección biselada, que hacía de cabezal. Fue una solución a la que el mismo arquitecto recurrió, al cabo de 6 años, en las 9 torres del Polígono de Capuchinos, Errenteria (70-ER).

\subsubsection{Solución D: la plaqueta como recurso compositivo}

Fue una solución empleada en los últimos años del ciclo, que consistía en revestir elementos superficiales de hormigón, como antepechos de balcones, petos de cubierta y otros, mediante la adhesión de plaquetas de ladrillo o ladrilletas que imitaban al ladrillo caravista. El uso de estas plaquetas se impuso a medida que las empresas fabricantes provenientes de regiones de fuerte tradición cerámica, como el Levante o Castilla, consolidaban sus redes comerciales en el País Vasco. Véase a este respecto el caso 73-ER, que presenta una com- 
posición a base de paños de ladrillo caravista dispuesto a sogas, y de otra serie de paños que recubren los antepechos de hormigón curvos, mediante plaquetas dispuestas a sardinel y a junta vertical corrida, poniendo en evidencia el carácter no estructural del paño en cuestión (ver figura 5).

\subsubsection{Solución $\mathrm{E}$ : la falsa fábrica}

La última solución consistía en ocultar completamente los elementos estructurales. Con tal fin, el ladrillo caravista era recortado mediante sierra de disco, en función del elemento

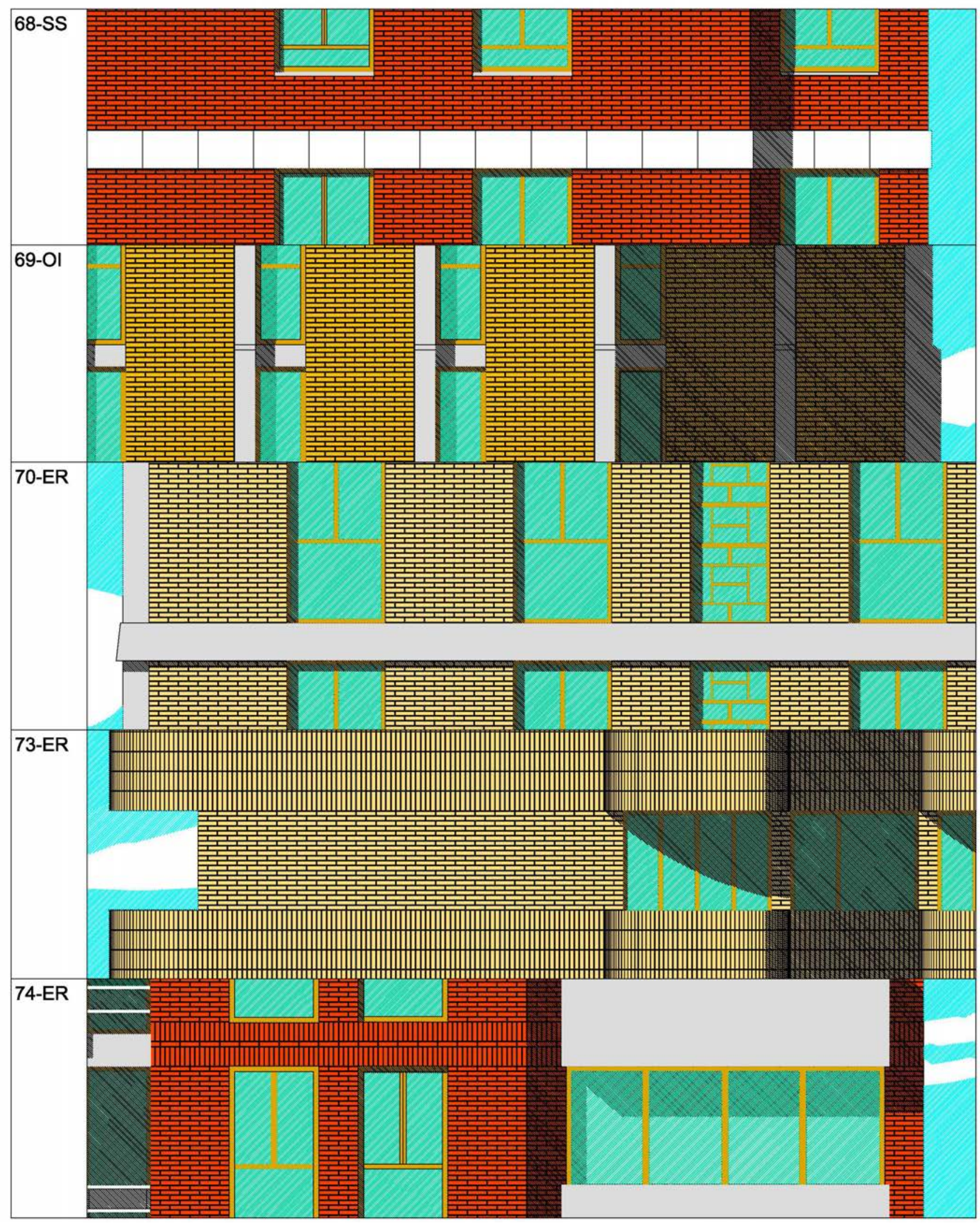

Figura 5. Composiciones originales recientemente ocultadas como consecuencia de la rehabilitación (2015-17). 
que fuera a recubrir: postes, cantos de forjado o pantallas. Los cantos de forjado podían ser revestidos con ladrillo recortado dispuesto a sogas, aparentando ser una fábrica portante (70-DE), o disponiendo dos hiladas a sardinel (68-AR), entre las cuales podía intercalarse una hilera de ladrillos dispuestos a sogas (74-ER). Los dinteles de los huecos quedaban resueltos con una hilera de ladrillos dispuestos a sardinel, y el revestimiento de los postes era resuelto sin recurrir a ninguna otra disposición ni aparejo.

\subsection{Ante la ocultación (conclusión)}

A comienzos de los años 6o, transcurridos quince años desde que las fachadas dejaran de ser portantes y se diera una disociación entre la estructura y los elementos de cerramiento, un nuevo tipo edificatorio rendía tributo a su propio sistema constructivo, que nacía precisamente como consecuencia de esta escisión. La evolución de los casos estudiados da fe de que dicho fenómeno se acentuó a medida que se consolidaba el nuevo tipo edificatorio, y que fue a finales de aquella década cuando se intensificó la participación del hormigón y del ladrillo caravista en la composición de las fachadas, dando vía a una serie de soluciones que no se reprodujeron en otros países del entorno, y que no volverían a ser empleadas tampoco en España.

Nada hay que objetar a la rehabilitación de estas envolventes que han terminado por demostrarse insuficientes ante la evolución experimentada por los estándares energéticos y los niveles de confort. No obstante, aunque sea en nombre de logros indiscutibles como el bienestar y el ahorro, hay que reconocer que la solución por la que el sector de la rehabilitación parece haberse decantado a la hora de rehabilitar este tipo residencial, va a incidir en la percepción de un elemento cuya vocación testimonial trasciende de lo meramente tecnológico, y que da cuenta con sinceridad de una época marcada por el crecimiento, la carencia de medios industrializados y el ingenio constructivo. Apórtense estas notas para poner de relieve las soluciones estudiadas, puesto que la intervención sobre un paramento mediante la adición de una nueva piel no deja de ser un acto de ocultación que altera su secuencia estratigráfica (28), y dado que la trascendencia del acto depende de lo elaborada que haya sido la interpretación del paramento original en términos artísticos, históricos o tecnológicos.

\section{REFERENCIAS}

(1) Plan Director de Vivienda 2013-2016 (2013). Departamento de Empleo y Políticas Sociales. Gobierno Vasco.

(2) Font, A. (1994). La Práctica del Planeamiento Urbanístico. En Moya, L. (Ed.), La práctica del urbanismo (pp. 25-53). Madrid: Síntesis.

(3) Vinuesa Angulo, J., Vidal Domínguez, M.J. (1991). Los procesos de Urbanización. Madrid: Síntesis.

(4) Etxepare, L., Lizundia, I., Sagarna, M., Uranga, E.J. (2015). Las torres de Bidebieta en San Sebastián (1962-1966). Un avance de altura para la construcción de la vivienda pública. En Huerta, S. (Ed.), Actas del Noveno Congreso Nacional y Primer Congreso Internacional Hispanoamericano de Historia de la construcción, Vol. 1 (pp. 585-592). Madrid: Instituto Juan de Herrera.

(5) Thornton, M. (2004). Who Predicted the Bubble? Who Predicted the Crash? The Independent Review, 9: 5-30.

(6) Galdós, R. (1991). De la expansión al estancamiento: una panorámica de la evolución demográfica del País Vasco. Boletín de la Asociación de Geógrafos Españoles, (13): 75-87.

(7) Rodríguez Marcos, J. (2001). Estructura y cambios del parque residencial de la Comunidad Autónoma de Euskadi. Eustat, Instituto Vasco de Estadística.

(8) Heckmann, O., Schneider, F. (2011). Floor Plan Manual Housing. Berlin: Birkhäuser.

(9) Plumridge, A., Meulenkanp, W. (2000). Brickwork: architecture and design, pp. 161-203, Londres: Phoenix Illustrated.

(10) Kurtz, F., Monzón, M., López-Mesa, B. (2015). Obsolescencia energética y acústica de la vivienda social de la postguerra española en áreas urbanas vulnerables. El caso de Zaragoza. Informes de la Construcción, 67(EXTRA-1): mo21, doi: https://doi.org/10.3989/ic.14.062.

(11) Cabrero, F. (1955). Viviendas económicas en Madrid. Informes de la Construcción, 7(70).

(12) Ross, K. (2002). Non-traditional housing in the UK - A brief review, Londres: The Council of Mortgage Lenders.

(13) Pourghazian, H. (2008). Industrial Construction Methods for Cost-Effective and Energy-Efficient Multi-Storey Buildings, p. 36, Estocolmo: KTH, Royal Institute of Technology.

(14) Del Águila, A. (1986). Las tecnologías de la industrialización de los edificios de vivienda, p. 97, Madrid: Colegio Oficial de Arquitectos de Madrid.

(15) La construction préfabriquée en Europe (1958). Paris: Agence Européenne de productivité e l'organisation européenne de coopération économique.

(16) Bestraten, S., Hormias, E., Domínguez, M. (2015). Bellvitge 50 años. Historia de un barrio de L’Hospitalet. Centre d'Estudis de L'Hospitalet.

(17) Bilbao, L. (2006). Algunas consideraciones sobre la historia de la industrialización de la construcción de viviendas durante el Desarrollismo (1960-1975): la aportación bilbaína al debate de la industrialización de la vivienda. Informes de la Construcción, 58(502), doi: https://doi.org/10.3989/ic.2006.v58.i502.388

(18) Sambricio, C. (2012). Cuando la vanguardia arquitectónica fue construcción. En Macías, M.C. (Coord.), Rafael Leoz, arquitecto de la Embajada de España en Brasil (pp. 22-35). Brasilia: Embajada de España en Brasil.

(19) Adell Argilés, J.M. (200o). Arquitectura sin fisuras, pp. 21-33, Madrid: Munilla-Lería.

(20) Domínguez Amarillo, S., Sendra Salas, J.J., Oteiza San José, I. (2016). La envolvente térmica de la vivienda social. El caso de Sevilla, 1939 a 1979. Madrid: Instituto de Ciencias de la Construcción Eduardo Torroja.

(21) Informe sobre la situación del sector de la vivienda en España (2010). Ministerio de la Vivienda España.

(22) Etxepare, L., Lizundia, I., Sagarna, M., Uranga, E.J., Malles, E., Ibarloza, E.M., Ibarloza, A. (2016). Estudio del impacto en el coste, debido a las nuevas exigencias normativas, de la rehabilitación de la vivienda colectiva construida entre 
1960 y 1980 en el País Vasco y sus consecuencias económicas y sociales. Proyecto de Investigación EHU14/56. Vicerrectorado de Investigación Universidad del País Vasco - Euskal Herriko Unibertsitatea.

(23) Lizundia-Uranga, I. (2015). La solución de fachada convencional del periodo desarrollista en el caso de Gipuzkoa: declive (y final) de un sistema constructivo. Informes de la Construcción, 67(538): e079, doi: https://doi.org/10.3989/ic.13.167

(24) Gössel, P., Leuthäuser, G. (2001). Arquitectura del siglo XX, 448 pp., Köln: Taschen.

(25) Bozal Fernández, V. (1996). Historia de las ideas estéticas y de las teorías artísticas contemporáneas, p. 384, Madrid: Visor.

(26) Ruiz Cabrero, G. (1989). Spagna. Architettura 1965-1988, p. 58, Milan: Electa.

(27) Fernandez de Betoño, U. (2010). Iñaki Galarragarekin solasean: Arkitekturaren aldeko aldartea sortu nahi izan genuen. Aldiri (3): 14-15.

(28) Harris, E. (1979). Principles of Archaeological Stratigraphy. San Diego: Academic Press Inc. 